\title{
Open Questions
}

\author{
G.G. Ross \\ Rudolf Peierls Centre for Theoretical Physics, \\ Department of Physics, \\ University of Oxford, \\ 1Keble Road, \\ Oxford, \\ England
}

\begin{abstract}
As we enter the LHC era the open questions in hard processes shift to the prospects for discovery of physics beyond the Standard Model. In this talk I discuss the main phenomenological questions that will need to be answered if we are to be able to distinguish between the various suggestions for the nature of the new physics that may be visible at the LHC.
\end{abstract}

\section{Introduction.}

In thinking about an appropriate choice for a talk on "Open Questions" I was influenced by the fact that the LHC era is almost upon us and that it is time to think seriously about the questions that will need to be answered if the LHC is to reveal the nature of the physics "Beyond the Standard Model" that many think must be present if we are to answer the questions left unanswered by the Standard Model (SM).

The LHC will open a new energy frontier and should reveal significant information about what lies Beyond the Standard Model. The fact that the electroweak breaking scale is well within the reach of the LHC means that we will be able to probe the origin of mass. In particular we should be able to answer the first open question whether the spontaneous symmetry breaking responsible for the masses of the weak gauge bosons and the quark and lepton current masses is due to an elementary Higgs boson, as in the Standard Model, or involves a composite Higgs bound by a new strong interaction alternatives as in technicolour. The need to solve the hierarchy problem which arises because radiative corrections tend to drive up the electroweak breaking to the scale of new physics strongly suggests that signals for new physics will appear at an energy scale less than $1 T e v$ and within the reach of the LHC.

A second open question is whether the fundamental forces are unified, possible at a very high scale. The precision with which the gauge couplings unify is remarkable, accurate to better than $1 \%$, and provides the best quantitative indication we have for such a unification. However this precision only applies if the effective theory at a scale of $1 T e v$ is supersymmetric, consistent with the constraint following from the supersymmetric solution to the hierarchy problem. The new supersymmetric partners of the Standard Model states should be within the reach of the LHC but, even if found, it will require considerable effort to establish their supersymmetric origin.

In seeking a unified theory it is natural to include gravity and the fact that the gauge coupling unification scale is close to the Planck scale is some indication of this unification. The best candidate for a quantum theory of gravity is the superstring and a further open question is whether there will be evidence for strings at the LHC. If the string states are at the Planck scale such indications will necessarily be indirect, leaving relations amongst the parameters of the effective low energy theory such as the relations between the gauge 
couplings. For the case of low energy supersymmetry the superstring unification may give relations between the soft supersymmetry breaking parameters, such as the squark and slepton masses. Another possibility, which has been suggested as an alternative solution to the hierarchy problem, is that new states associated with the additional space dimensions required by the string, or indeed the string states themselves, are associated with a low scale of $O(1 \mathrm{TeV})$ and are accessible to discovery by the LHC. If such states are present it will require much work to distinguish them from other possibilities such as a supersymmetric origin.

Another important open question is the origin of dark matter. Many of the suggestions for solving the hierarchy problem involve dark matter candidates; for example the lightest supersymmetric particle (LSP) or the lightest KK mode associated with a new space dimension. To prove that any of these candidates is the source of dark matter will require that its detailed interactions are determined in order to reliably compute the annihilation cross sections and hence the relic abundance.

Given these questions what are the likely LHC answers? The search for new states largely relies on missing momentum signals putting the questions firmly in the area of expertise covered by this meeting and I will discuss some of the suggestions that have been made for ways to determine the underlying physics origin of any new states found at the LHC. In this talk I will start by considering the (pessimistic) possibility that the electroweak breaking is associated with a Higgless model or a very heavy Higgs model. Such possibilities occur in models with large extra dimensions or in technicolour models and can be very difficult to test at the LHC. The second half of the talk will concentrate on the new physics associated with a light Higgs boson, for example in supersymmetric theories or in little Higgs models.

\section{Higgless (or very heavy Higgs) at the LHC.}

The most pessimistic possibility for LHC physics is that the Standard Model is all there is up to the limit of the LHC reach with a very heavy Higgs scalar or even no Higgs scalar at all. Precision measurements at LEP argue against this possibility because they favour the radiative corrections corresponding to a light Higgs[2]. However new physics at the TeV scale could affect these corrections so a heavy Higgs is still a possibility. In perturbation theory one may show that a Standard Model Higgs cannot be very heavy without violating perturbative unitarity. The effect is strongest in processes involving the longitudinal component of the $\mathrm{W}$ and $\mathrm{Z}$ bosons. For example in the Standard Model the graphs (a) to (d) of Fig 1 are the tree level contributions to $W_{L}+Z_{L}$ elastic scattering. If the Higgs is very heavy the graph (d) is suppressed. In this case the remaining graphs will violate the unitarity bound at a scale $\Lambda \simeq 1.8 T e V$. Of course this does not mean that unitarity is violated but it does require that higher order graphs restore unitarity, i.e. the interaction becomes strong. Recently an alternative class of "Higgsless" models have been constructed which postpone the scale at which the interactions become strong[4]. They are based on models with additional space dimensions and in the four dimensional effective field theory there are the additional contributions of graphs (e) and (f) involving the exchange of massive vector bosons, Kaluza Klein excitations of the $W$. The effect of these contributions is to raise the scale at which the unitarity bound is reached to $\Lambda \simeq 5-10 \mathrm{TeV}$, out of the reach of the LHC. What are the prospects for measuring at the LHC the strong coupling behaviour in the heavy Higgs case or the presence of the additional heavy vector bosons in the Higgsless case? From Fig 1 one may see that in the SM case there is no s-channel resonance while in the Higgsless case there 


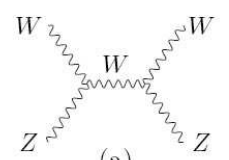

(a)

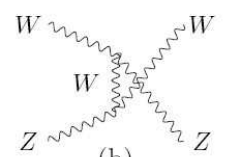

(b)

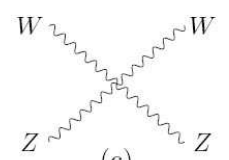

(c)

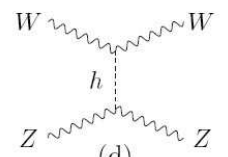

(d)

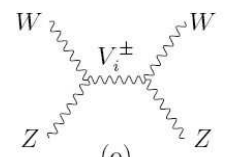

(e)

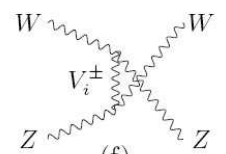

(f)

Figure 1: Feynman graphs contributing to $W_{L}+Z_{L}$ elastic scattering.

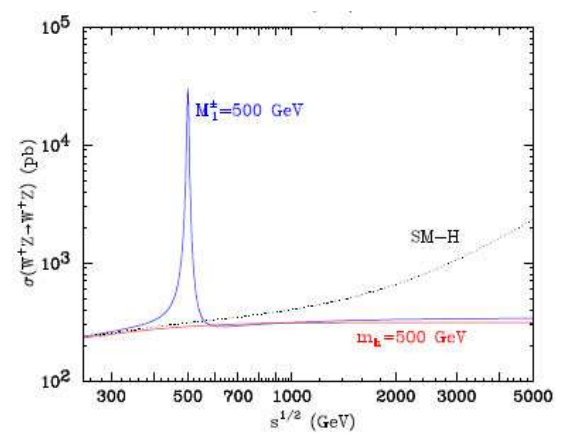

Figure 2: $W_{L}+Z_{L}$ elastic scattering (i) in the Standard Model without a Higgs boson (ii) in the Standard Model with a $500 \mathrm{GeV}$ Higgs and (iii) in the Higgsless model with a $500 \mathrm{GeV}$ heavy vector boson.

is a characteristic narrow resonance. In the SM case when the weak interactions become strong there may be a s-channel resonance due to strong binding effects but it is expected to be very wide and heavy, approximately $2 \mathrm{TeV}$ if the strong interaction is QCD-like. For this reason the $W_{L}+Z_{L}$ elastic scattering case provides a very good discriminator for the Higgsless models.

The production of the initial $W$ and $Z$ is via bremsstrahlung off quarks, and the signal is two forward jets plus a gauge boson pair giving a gold plated final state of two jets plus 3 leptons plus missing transverse momentum. The various cross sections are shown in Fig 2.

Figure 3 shows the number of events at the LHC to be expected in the SM with a $500 \mathrm{GeV}$ Higgs and in the Higgsless model for a heavy vector of mass $700 \mathrm{GeV}$ for an integrated luminosity of $300 \mathrm{fb}^{-1}[15]$. In addition are shown two estimates for the cross section for the case the Higgs mass is well above the perturbative limit corresponding to the case the weak interactions become strong at high energies. In this case it is necessary to include higher order radiative corrections to preserve unitarity and this has been done in two ways, the first using the $K$-matrix approach [5] and the second using Pade approximants [6]. The most obvious feature of these graphs is that in all cases the signal is very small for the SM with a Higgs of $500 \mathrm{GeV}$ or above. Given this small SM background the discovery reach for the Higgsless model requires only of the order of 10 events corresponding to $10 \mathrm{fb}^{-1}$ of data. By looking at various channels it is in principle possible to check this identification. In the SM the Higgs can appear as a resonance in the $W W \rightarrow W W, Z Z$ channels but not as we 


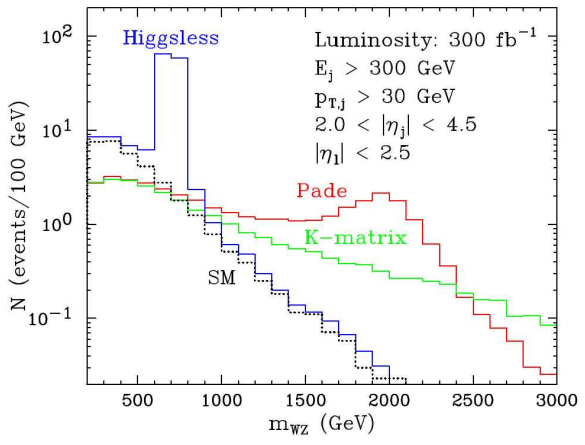

Figure 3: The number of events at the LHC for an integrated luminosity of $300 \mathrm{fb}^{-1}$ with the cuts indicated.

have seen in the $W Z \rightarrow W Z$ channel. For the case of the Higgsless models the resonance appears in the $W W \rightarrow W W$ and $W Z \rightarrow W Z$ channels but not in the $W W \rightarrow Z Z$ channel.

For the very heavy Higgs case it is necessary to establish the corresponding existence of strong interactions. However this requires the measurement of the excess of events of the curves labeled K-matrix and Pade over the SM case. The total number of such events for $300 \mathrm{fb}^{-1}$ is only 6 so the LHC will not be able to probe this possibility and it will be necessary to wait for the SLHC upgrade with a luminosity increase of a factor of 10 before one can measure the effect of a strongly interacting weak sector in the very heavy Higgs limit. This will still be very difficult and will require a detailed understanding of the initial parton distributions.

\section{Light Higgs and the hierarchy problem.}

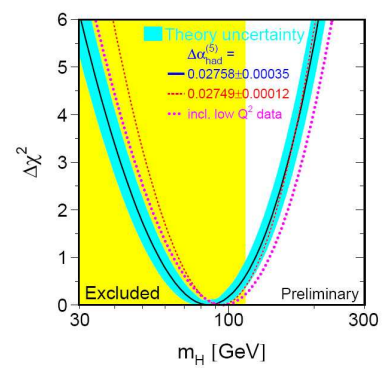

Figure 4: The value of $\Delta \chi^{2}$ as a function of the Higgs mass for a fit to precision observables in the Standard Model[2]

However there is already evidence that just the SM with a very heavy Higgs is unlikely. The evidence comes from the need to have a light Higgs to fit the precision electroweak data from LEP. In Fig 4 the $\chi$ squared dependence of the precision electroweak fit to the Higgs 
mass is shown [2] and corresponds to a bound on the Higgs mass given by $m_{H}<200 \mathrm{GeV}$. Given this I turn now to a discussion of the important questions that will need to be answered in the light Higgs case. The driving force in constructing extensions of the Standard model has been the requirement that it should solve the hierarchy problem, that is the need to control radiative corrections to the Higgs boson mass that would normally drive it to the scale of new physics such as the Grand Unified (GUT) scale or the Planck scale. As illustrated in Fig 5 there are two options - the Higgs could be composite or elementary.

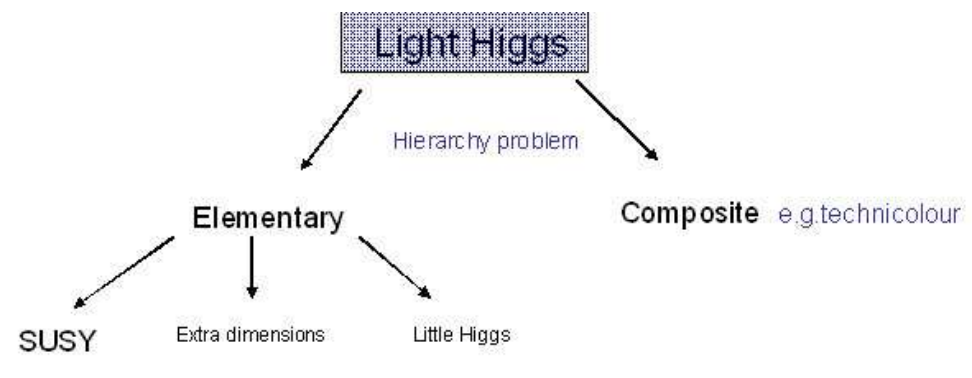

Figure 5: Competing explanations for the existence of a light Higgs scalar and the solution of the herarchy problem.

In the former case the hierarchy problem is evaded if the scale of compositeness is low, of $O(1 T e V)$, because the radiative corrections are cut-off at virtual momenta of order the composite scale. The achetypical example of a composite theory is technicolour. The need to suppress flavour changing neutral currents and the need to be consistent with precision electroweak tests have imposed strong constraints on technicolour showing that the strong binding force must be quite different from QCD. The difficulty associated with calculating strong interaction effects in such a theory makes it difficult to extract definite predictions but there should be a zoo of composite bound states including many familon states, pseudo Goldstone modes associated with the breaking of approximate family symmetries of the extended technicolour model. Given the difficulty in making definite predictions I will not discuss this possibility further here but refer the reader to a study of the signals to be expected in some particular models and references therein[8].

For the case the Higgs is elementary perturbative calculations may be applicable, making it possible to make more definite predictions for the signals to be expected at the LHC. This is the case if supersymmetry is responsible for protecting the Higgs against large radiative corrections to its mass[9]. Moreover the new supersymmetric states must be light, accessible to production and detection at the LHC, if the hierarchy problem is to be avoided. While many of the properties of the supersymmetric states are determined, being related to the properties of their Standard Model partners, the spectrum results from supersymmetry breaking and consequently is less well understood. In Fig 6 I show a variety of supersymmetric spectra for various possible supersymmetry breaking mechanisms[10]. It is clear that there are a wide variety of possibilities and one of the important tasks we need to address is to identify the best ways to measure the mass spectra at the LHC. This will require the development of techniques to measure both the masses and spins of the new states for the case that the signals always involve missing momentum. This need for spin measurement is particularly important because alternative explanations of the hierarchy problem can give 


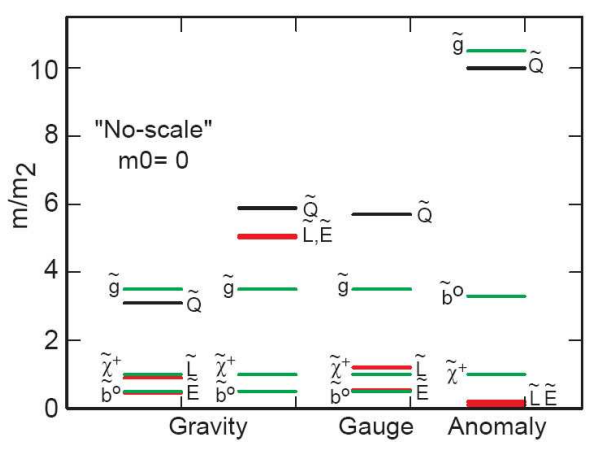

Figure 6: The supersymmetric spectrum for various choices of supersymmetry breakingmechanisms [10].

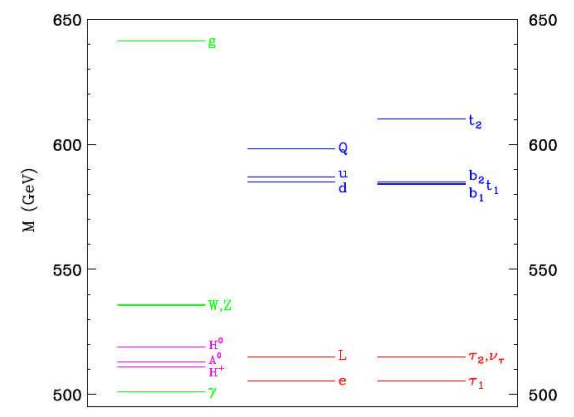

Figure 7: One loop corrected mass spectrum of the first KK level in MUED for a characteristic choice of parameters[11].

similar spectra. This is illustrated in Fig 7 [11] where one can see significant similarities in the mass spectrum coming from an extra dimensional theory with Universal Extra Dimensions (UED)[12]. The reason for this is that the radiative corrections responsible for splitting the states are similar simply because the gauge interactions responsible for much of the splitting are the same. In the UED case the new states are the KK excitations of the SM states while in the SUSY case the new states are the SUSY partners of the SM states; in both cases they belong to the same representations of the SM gauge group as their SM partners. Of course in the case of UED there will be a tower of KK excitations but the splitting may be such tat only the first excitations are accessible to the LHC. However the spins of the states differ in the SUSY and UED cases so the spin determination is crucial to the identification of the nature of the new physics. There are more reasons why measuring the spectrum in detail is important. In the case of supersymmetry the mass spectrum offers a window on the high energy theory and on the possible unification of the fundamental forces. This illustrated in Fig 8 where one may see that, like the gauge couplings, the soft SUSY masses have radiative corrections that split them even if they are universal at a high 
scale $^{\mathrm{a}}$. As may be seen in the first panel the gauge couplings unify very precisely at a scale of $2.10^{6} \mathrm{GeV}$ [13] due to the inclusion of the supersymmetric states in the calculation of the corrections. It is largely for this reason that so much attention has been paid to the supersymmetric solution of the hierarchy problem. However given that the value of the unification coupling at the unification scale is unknown as is the unification scale itself there is only one prediction being tested by the gauge coupling unification and it would be very important if further evidence of unification is found. Such evidence could be provided by the unification of soft SUSY breaking masses as is illustrated in the second panel of Figure 8. The evidence for unification will be overwhelming if the masses as measured at low scales should follow the pattern shown which follows if the masses unify at the same scale as is found for the gauge coupling unification. In fact there is already some evidence for soft mass unification because the Higgs scalars are also expected to unify. Due to the large top Yukawa coupling radiative corrections systematically drive the mass squared of the Higgs negative, triggering electroweak breaking[14]. The top squarks, which are the other scalar states to feel the effects of the top Yukawa coupling, are coloured and hence have stabilising QCD radiative corrections which drive the mass squared positive and dominate over the Yukawa contribution. Thus mass unification leads to a natural explanation of why the Standard Model gauge group breaks to $S U(3) \times U(1)$.
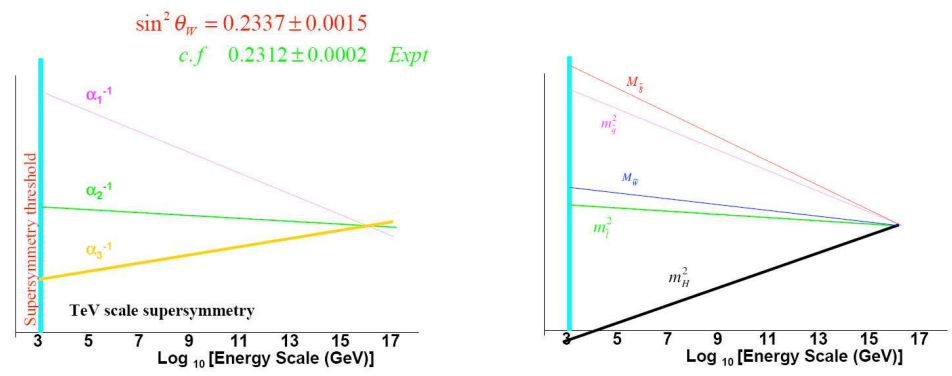

Figure 8: Renormalisation group flow of a) gauge couplings and b) scalar masses, with unification at a high scale.

Another very important reason for measuring the spectrum and spin structure of the new supersymmetric states is that only with this information will it be possible to determine whether the lightest of such states (LSP) provides the answer to the dark matter problem[9]. The dark matter abundance is very sensitive to the LSP mass and the states to which it couples, usually the sleptons, so it is particularly important to be able to measure them.

Given this motivation what are the prospects for being able to measure the masses of any new states, given that identification of such states requires large missing momentum? As a good example let us consider the possibility for slepton mass measurement. Direct slepton production has large SM corrections due to $W W$ and $\bar{t} t$ production. For this reason a more promising channel is production of neutralinos, $\chi_{2}$, followed by cascade decay to the LSP,

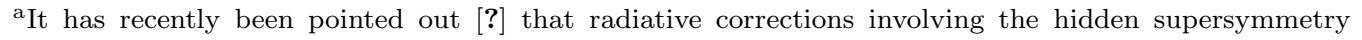
breaking sector can significantly change the evolution of the soft scalar masses and obscure the unification of masses. In many of the favoured SUSY breaking schemes these corrections are small and in any case the unification of gaugino masses is unaffected by such corrections.
} 
$\chi_{1}$, which is sensitive to the slepton mass. The relevant Feynman diagrams are shown in Fig 9. For the case that the decay occurs through a real $Z$ this contribution dominates
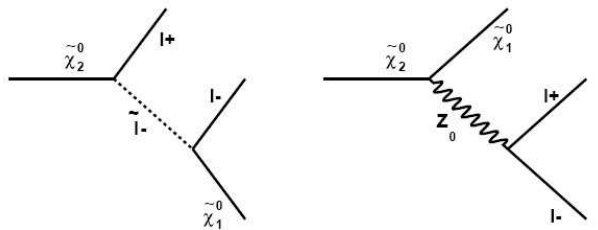

Figure 9: Feynman diagrams contributing to neutraino decay.

and there is no mass information. For the case the decay is through a virtual intermediate state the dilepton invariant mass distribution endpoint is given by

$$
m_{l l}=m_{\chi_{2}}-m_{\chi_{1}}
$$

If the decay is through a real slepton the end point is

$$
m_{l l}=\sqrt{\frac{\left(m_{\chi_{2}}^{2}-m_{l}^{2}\right)\left(m_{l}^{2}-m_{\chi_{1}}^{2}\right)}{m_{l}^{2}}}
$$

Thus endpoint measurement can give one constraint on the SUSY mass spectrum. The full invariant mass distribution gives some further information which can establish reasonable upper and lower bounds on light slepton masses. A detailed study including detector effects demonstrated that the signal can be distinguished from the background at the LHC with $10 \mathrm{fb}^{-1}[?]$. While the information thus obtained is important, it falls short of a complete determination of the light neutralino and slepton spectrum. To obtain more information requires studying additional processes, for example cascade decays involving longer chains and additional final states. This gives more information because there are now several invariant masses that can be measured and used to determine the masses of the particles involved in the chain. However to access this information it will be necessary to fold in the detector and background effects together with the ambiguities associated with misidentification of the particle in the cascade chain that necessarily will be present in the multiparticle final states that abound at the LHC. Doing this and identifying the most sensitive processes for determining the mass spectrum remains one of the pressing open questions. Clearly, since all such processes necessarily involves high transverse missing momenta in order to avoid SM backgrounds, the expertise of calculating DIS processes will be crucial in this task.

An even more difficult task will be the development of efficient methods capable of measuring the spin of the new particles; as emphasised above this is crucial to the identification of the nature of the physics beyond the Standard Model. One promising method again involves studying the invariant mass distributions associated with long cascade chains. Consider the decay chain of Fig 10 which leads to a quark jet and a dilepton plus missing energy signal. The lower state assignments correspond to the states involved in UED in the decay of a KK quark excitation. The upper state assignments correspond to the states involved in SUSY in the decay of a squark. The spins of the states in the decay chain for the UED case are $1 / 2,1$, 
$1 / 2$ and 1 while for the SUSY case they are $0,1 / 2,0.1 / 2$. The question is can one distinguish these two possibilities? In general there are in fact six possible spin assignments giving rise

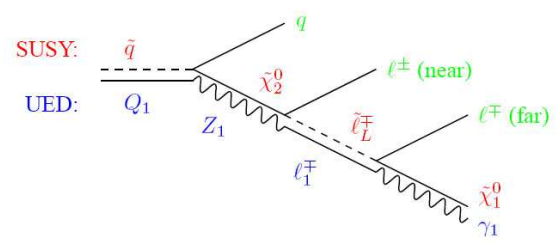

Figure 10: Cascade decays for a KK quark excitation in a UED model and for a squark decay in a SUSY model.

to the same visible final state which can arise in SUSY and UED models. These are shown in Fig 11. A discriminant that can differentiate between these cases is given by[16, 17]

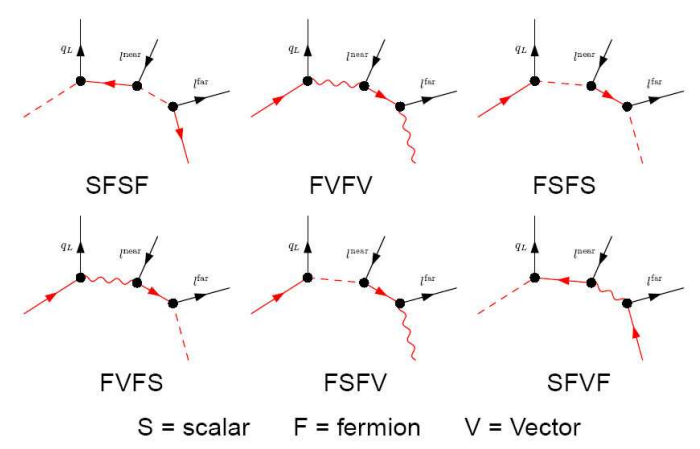

Figure 11: Six possible spin assignments giving rise to a quark jet plus a charged lepton antilepton pair.

$$
\frac{d P}{d m}=\frac{1}{\Gamma} \frac{d \Gamma}{d m}
$$

where $\Gamma$ is the total decay rate of the chain and $m_{a b}^{2}=\left(p_{a}+p_{b}\right)^{2}$ is the invariant mass squared of particles $a$ and $b$. For example, consider the invariant mass distribution of the lepton pair. Consider also a choice of masses given by the benchmark SPS1a for the SUSY case and by inverse radius $R^{-1}=800 \mathrm{GeV}$ and higher dimension Planck mass $\Lambda$ given by $\Lambda R=20$ for the UED case. For these choices the distribution for the two possibilities given in Fig 11 are shown in Figure 12[17]. One may see that the distributions do distinguish between several of the possible spin chains. To quantify this the authors of [17] determined the number of events that are needed if the SUSY decay chain of Fig 11 is true to establish that it is 1000 times more likely than each of the other possibilities. This is shown by the light gray entry in Fig 13. While the numbers are encouragingly small for this case some caveats are in order. In particular in a multiparticle final state there will be ambiguities 

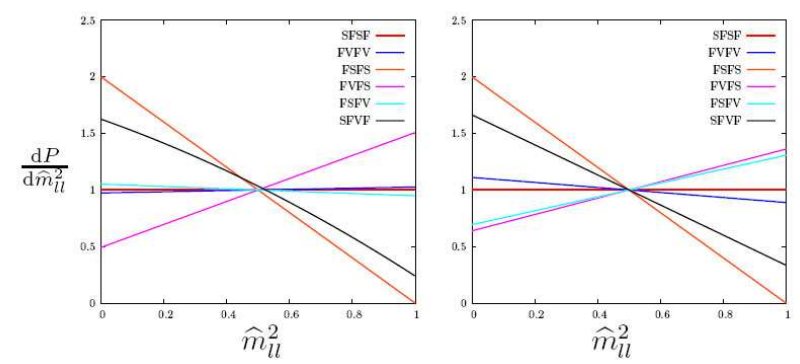

Figure 12: The $m_{l l}^{2}$ distribution for SUSY and UED spin chains.

\begin{tabular}{|r|rrrrrr|}
\hline $\mathrm{T} \downarrow \mathrm{S} \rightarrow$ & SFSF & FVFV & FSFS & FVFS & FSFV & SFVF \\
\hline SFSF & $\infty$ & 60486 & 23 & 148 & 15608 & 66 \\
FVFV & 60622 & $\infty$ & 22 & 164 & 6866 & 62 \\
FSFS & 36 & 34 & $\infty$ & 16 & 39 & 266 \\
FVFS & 156 & 173 & 11 & $\infty$ & 130 & 24 \\
FSFV & 15600 & 6864 & 25 & 122 & $\infty$ & 76 \\
SFVF & 78 & 73 & 187 & 27 & 90 & $\infty$ \\
\hline
\end{tabular}

Figure 13: The number events needed to establish that a given spin chain assignment is 1000 tme more likely than the other possibilities. The light entry corresponds to the case that the SUSY case of Fig 11 is true.

in identifying the relevant lepton momenta when forming the invariant mass. Furthermore the discriminant is dependent on the masses within the cascade and these masses may be poorly determined (the examples above assumed definite, but not identical, mass spectra for the SUSY and UED cases). Indeed, as emphasised by [18], one may adjust the parameters of the UED model so that the mass spectra actually coincides with the SUSY case and then the lepton mass squared distribution are so similar for the SUSY and UED cases that they are not be distinguishable once the background, radiative corrections and the detector simulation are taken into account. The authors of reference [18] studied in some detail the jet-lepton invariant mass distribution as a way to resolve this ambiguity. The spin information comes from the asymmetry between the positive and negative charged lepton pairings with the quark jet. The result is shown in Fig 16. The errors are calculated assuming $10 \mathrm{fb}^{-1}$ luminosity and a $10 \%$ jet energy resolution. One may see that it is possible to distinguish the SUSY chain from the UED chain. However no account has been taken yet of the uncertainty associated with misidentifying the jets. Furthermore it relies on a charge asymmetry which may be very small if the initial production mechanism is dominated by gluons.

In summary, the LHC will probe the energy regime relevant to electroweak breaking and should shed light on the origin of mass. Many possibilities have been identified for the 


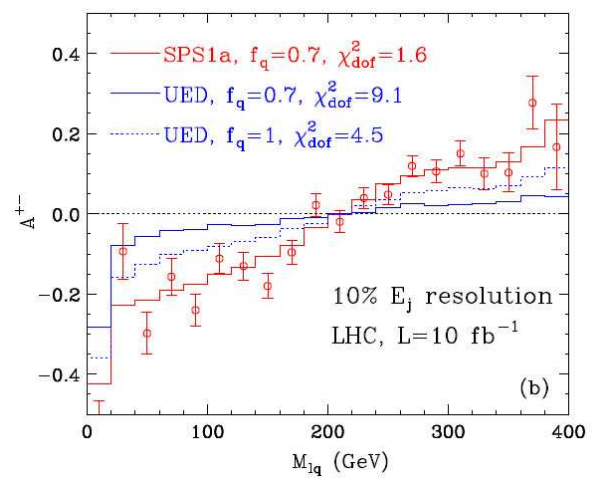

Figure 14: The lepton-jet charge asymmetry plotted versus the lepton-jet invariant mass for the case of SUSY and UED disucssed in the text. The quantity $f_{q}$ gives the proportion of squarks or KK quarks compared to the antiparticles.

physics Beyond the Standard Model relevant to the LHC, mostly motivated by the need to avoid the hierarchy problem. What has become clear is that it will need detailed information on the masses, spins and interactions to distinguish between these possibilities. Searches for new states rely on missing momentum signals to separate them from Standard Model backgrounds and so the expertise developed for studying perturbative processes in Deep Inelastic Scattering will be invaluable in the analysis of these signals. Obviously the parton distribution functions at the LHC will be an essential input to the calculation of these signals. So too will be the calculation of higher order radiative corrections for processes involving massive states preliminary studies as we know these can significantly modify the cross sections. In addition it is very important to develop precise methods to determine the mass and spin of new states. preliminary work has been carried out studying the possibility of measuring masses and spins in cascade decay chains. The results suggest that while mass and spin discriminants based on invariant mass distributions are viable they are going to be very difficult to achieve due to the many uncertainties associated with the overall rate, the backgrounds, the particle identification and the detector simulations. Alternative methods are being developed which may offer complementary information based on studying the dependence of the cross section on angular variables[19]. Clearly much work remains to be done in establishing the optimum methods and in doing a complete Monte Carlo simulation of the events.

\section{References}

[1] Slides: http: //indico. cern. ch/contributionDisplay $\cdot$ py? contribId=21\&sessionId=2\&conf Id=9499

[2] J. Alcaraz et al. [ALEPH Collaboration], arXiv:hep-ex/0612034.

[3] See M. S. Chanowitz, "The no-Higgs signal: Strong W W scattering at the LHC," and references therein, Czech. J. Phys. 55 (2005) B45 [arXiv:hep-ph/0412203].

[4] R. Sekhar Chivukula, D. A. Dicus and H. J. He, Phys. Lett. B 525 (2002) 175 [arXiv:hep-ph/0111016].

[5] A. Dobado, M. J. Herrero and J. Terron, Z. Phys. C 50, 205 (1991). 
[6] A. Dobado, M. J. Herrero and J. Terron, Z. Phys. C 50, 465 (1991).

[7] A.Birkedal, K.Matchev and M. Perelstein "Phenomenology of Higgsless models at the LHC" in "Tevatron-for-LHC report: Preparations for discoveries," eds. V. Buescher, M. Carena, B. Dobrescu, S. Mrenna, D. Rainwater and M. Schmitt, arXiv:hep-ph/0608322.

[8] K.Lane, "Search for low scale Technicolor at the Tevatron" in "Tevatron-for-LHC report: Preparations for discoveries," eds. V. Buescher, M. Carena, B. Dobrescu, S. Mrenna, D. Rainwater and M. Schmitt, arXiv:hep-ph/0608322.

[9] For extensive reviews of SUSY see: H. P. Nilles, Phys. Rept. 110, 1 (1984); J. A. Bagger, arXiv:hepph/9604232; S. P. Martin, arXiv:hep-ph/9709356. ; D. Bailin and A. Love, "Supersymmetric gauge field theory and string theory," Institute of Physics publishing 1994; M. Drees, R. Godbole and P. Roy, "Theory and phenomenology of sparticles: An account of four-dimensional N=1 supersymmetry in high energy physics," Cambridge University Press 2007; H. Baer and X. Tata, "Weak scale supersymmetry: From superfields to scattering events," Cambridge University Press 2006; P.Binetruy, "Supersymmetry, Theory, Experiment and Cosmology", Oxford University Press 2006.

[10] M. E. Peskin, arXiv:hep-ph/0002041.

[11] H. C. Cheng, K. T. Matchev and M. Schmaltz, Phys. Rev. D 66 (2002) 056006 [arXiv:hep-ph/0205314].

[12] T. Appelquist, H. C. Cheng and B. A. Dobrescu, Phys. Rev. D 64 (2001) 035002 [arXiv:hep$\mathrm{ph} / 0012100]$

[13] See D. M. Ghilencea and G. G. Ross, Nucl. Phys. B 606 (2001) 101 and references therein [arXiv:hep$\mathrm{ph} / 0102306]$.

[14] For a recent review see: L. E. Ibanez and G. G. Ross, arXiv:hep-ph/0702046.

[15] A.Birkedal, C.Group and K.Matchev, "Slepton mass measurements at the LHC" in "Tevatron-forLHC report: Preparations for discoveries," eds. V. Buescher, M. Carena, B. Dobrescu, S. Mrenna, D. Rainwater and M. Schmitt, arXiv:hep-ph/0608322.

[16] A. J. Barr, Phys. Lett. B 596 (2004) 205 [arXiv:hep-ph/0405052].

[17] C. Athanasiou, C. G. Lester, J. M. Smillie and B. R. Webber, JHEP 0608 (2006) 055 [arXiv:hep$\mathrm{ph} / 0605286]$.

[18] AK.Datta, K.Kong and K.Matchev, "Spin determination at the LHC" in "Tevatron-for-LHC report: Preparations for discoveries," eds. V. Buescher, M. Carena, B. Dobrescu, S. Mrenna, D. Rainwater and M. Schmitt, arXiv:hep-ph/0608322.

[19] A. J. Barr, JHEP 0602 (2006) 042 [arXiv:hep-ph/0511115] 